\title{
IDENTIFICATION PROBLEM FOR A WAVE EQUATION VIA OPTIMAL CONTROL
}

\author{
Suzanne Lenhart and Min Liang \\ University of Tennessee \\ Mathematics Department \\ Knoxville, TN 37996-1300 \\ Vladimir Protopopescu \\ Oak Ridge National Laboratory \\ Computer Science and Mathematics Division \\ Oak Ridge, TN 37831-6364
}

\begin{abstract}
We approximate an identification problem by applying optimal control techniques to a Tikhonov's regularization. We seek to identify the dispersive coefficient in a wave equation and allow for the case of error or uncertainty in the observations used for the identification.
\end{abstract}

\section{INTRODUCTION}

We apply optimal control techniques to find approximate solutions to an identification problem for a wave equation. Consider the wave equation in $Q=$ $\Omega \times(0, T):$

$$
\begin{aligned}
w_{t t} & =\Delta w+h w+f & & \text { in } Q \\
w & =0 & & \text { on } \partial \Omega \times(0, T) \\
w & =w_{0}(x) & & t=0, \quad x \in \Omega \\
w_{t} & =w_{1}(x) & & t=0, \quad x \in \Omega
\end{aligned}
$$

where $f \in L^{2}(Q), w_{0} \in H_{0}^{1}(\Omega), w_{1} \in L^{2}(\Omega)$ and $\Omega \subset R^{n}$ with $C^{2}$ boundary. We seek to identify the dispersive coefficient $h$ from observations of the solution on a set $Q^{\prime} \subset Q$.

The identification problem is to find bounded $h$ such that the corresponding solution $w=w(h)$ to (1.1) is close to the observations $z$ on $Q^{\prime}$. Thus, we want 
to minimize the functional

$$
J(h)=\frac{1}{2} \int_{Q^{\prime}}(w(h)-z)^{2} d x d t
$$

over the class

$$
U=\left\{h \in L^{\infty}(Q) \mid-M \leq h(x, t) \leq M\right\} .
$$

To approximate this problem, we introduce the following control problem for $\beta>0$ :

$$
\min _{h \in U} J_{\beta}(h)
$$

with

$$
J_{\beta}(h)=\frac{1}{2}\left(\int_{Q^{\prime}}(w(h)-z)^{2} d x d t+\beta \int_{Q} h^{2} d x d t\right) .
$$

The coefficient to be identified is viewed as a control, which is adjusted to get the corresponding solution, $w(h)$, close to the observations $z$. This type of approximation is called Tikhonov's regularization [1].

See Liang [6] for some further results on this type of control problem (without the connection to identification). See Lenhart, Protopopescu and Yong $[4,5]$ for similar approximation techniques for other wave equation problems. See $[1,2]$ for other approaches to using Tikhonov's regularization for identification problems.

In section 2, we prove the existence of an optimal control to the approximate problem (1.3) and characterize it through an optimality system. In section 3 , we connect these approximations to the identification problem as $\beta \rightarrow 0$. Specifically, we show that as $\beta \rightarrow 0$, the optimal control $h_{\beta}$ for the functional $J_{\beta}$, converges to a feasible coefficient. We allow for the possibility that there is some error or uncertainty in the observations $z$. Thus the solution, which is the limit of $w_{\beta}=w\left(h_{\beta}\right)$ as $\beta \rightarrow 0$, may not match the observation $z$.

\section{OPTIMAL CONTROL PROBLEM}

We assume $f, f_{t} \in L^{2}(Q)$ and define the bilinear form:

$$
B[u, v ; t]=\int_{\Omega} \nabla u \nabla v d x-\int_{\Omega} h u v d x .
$$

Definition: Given $h \in U$, a function $w \in L^{2}\left(0, T ; H_{0}^{1}(\Omega)\right)$, with $w_{t} \in$ $L^{2}\left(0, T ; L^{2}(\Omega)\right)$ and $w_{t t} \in L^{2}\left(0, T ; H^{-1}(\Omega)\right)$ is a weak solution of the problem (1.1) if

$$
\int_{0}^{T}\left\langle w_{t t}, \phi>d t+\int_{0}^{T} B[w, \phi ; t] d t=\int_{Q} f \phi d x d t\right.
$$


for any $\phi \in H_{0}^{1}(\Omega)$, and $w(0)=w_{0} ; w_{t}(0)=w_{1}$, Here $<,>$ denotes the duality between $H^{-1}(\Omega)$ and $H_{0}^{1}(\Omega)$.

Lemma 2.1 For $h \in U$, the problem (1.1) has a unique weak solution $w$ and

$$
\begin{aligned}
& \sup _{0 \leq t \leq T}\left(\|w(t)\|_{H_{0}^{1}(\Omega)}+\left\|w_{t}(t)\right\|_{L^{2}(\Omega)}\right)+\left\|w_{t t}\right\|_{L^{2}\left(0, T ; H^{-1}(\Omega)\right)} \\
\leq & C\left(\|f\|_{L^{2}(Q)}+\left\|w_{0}\right\|_{H_{0}^{1}(\Omega)}+\left\|w_{1}\right\|_{L^{2}(\Omega)}\right) .
\end{aligned}
$$

The proof uses standard estimates on Galerkin approximations starting from smooth initial data $[3,6]$.

Theorem 2.1: There exists an optimal control $h_{\beta} \in U$, which minimizes the objectve functional $J_{\beta}(h)$ over $h \in U$.

Proof: Let $\left\{h^{n}\right\} \in U$ be a minimizing sequence such that

$$
\lim _{n \rightarrow \infty} J\left(h^{n}\right)=\inf _{h \in U} J(h) .
$$

Lemma 2.1 gives a priori estimates on $w^{n}=w\left(h^{n}\right)$ with bounds independent of $n$. On a subsequence, by weak compactness, there exists $w_{\beta}$ in $L^{2}\left(0, T ; H_{0}^{1}(\Omega)\right)$ such that

$$
\begin{array}{r}
w^{n} \rightarrow w_{\beta} \text { weakly in } L^{2}\left(0, T ; H_{0}^{1}(\Omega)\right), \\
w_{t}^{n} \rightarrow\left(w_{\beta}\right)_{t} \text { weakly in } L^{2}(Q), \\
h^{n} \rightarrow h_{\beta} \text { weakly in } L^{2}(Q), \\
w_{t t}^{n} \rightarrow\left(w_{\beta}\right)_{t t} \text { weakly in } L^{2}\left(0, T ; H^{-1}(\Omega)\right) .
\end{array}
$$

By a compactness result [7], we have $w^{n} \rightarrow w^{\beta}$ strongly in $L^{2}(Q)$ and hence $w_{\beta}=w\left(h_{\beta}\right)$. Using the lower-semicontinuity of $L^{2}$ norm with respect to weak convergence, we conclude that $h_{\beta}$ is an optimal control.

To characterize the optimal control, we need to differentiate the maps

$$
h \rightarrow J_{\beta}(h) \text { and } h \rightarrow w(h) .
$$

Lemma 2.2: The mapping

$$
h \in U \longrightarrow w(h)
$$

is differentiable in the following sense;

$$
\frac{w(h+\varepsilon \ell)-w(h)}{\varepsilon} \rightarrow \Psi \text { weakly in } L^{2}\left(0, T ; H_{0}^{1}(\Omega)\right)
$$

as $\varepsilon \rightarrow 0$, for any $h, h+\varepsilon \ell \in U$. Moreover $\Psi$ is a weak solution of

$$
\begin{aligned}
\Psi_{t t} & =\Delta \Psi+h \Psi+\ell w, \quad \text { in } Q \\
\Psi & =0 \quad \text { on } \partial \Omega \times(0, T) \\
\Psi & =0 \quad t=0, x \in \Omega \\
\Psi_{t} & =0 \quad t=0, x \in \Omega
\end{aligned}
$$


where $w=w(h)$.

Proof: Denoting $w^{\varepsilon}=w(h+\varepsilon \ell)$ and $w=w(h)$, we have

$$
\begin{aligned}
& \sup _{0 \leq t \leq T}\left\|\frac{\left(w^{\varepsilon}-w\right)(t)}{\varepsilon}\right\|_{H_{0}^{1}(\Omega)}+\left\|\frac{w_{t t}^{\varepsilon}-w_{t t}}{\varepsilon}\right\|_{L^{2}\left([0, T] ; H^{-1}(\Omega)\right)} \\
& \leq \quad C\|\ell\|_{L^{\infty}} .
\end{aligned}
$$

On a subsequence, we have the existence of $\Psi$ in $L^{2}\left(0, T ; H_{0}^{1}(\Omega)\right)$ and $\Psi$ solves (2.1).

We derive the necessary conditions to characterize an optimal control.

Theorem 2.2: Given an optimal control, $h_{\beta}$, and corresponding state, $w_{\beta}=$ $w\left(h_{\beta}\right)$, there exists a weak solution $p$ in $L^{2}\left(0, T ; H_{0}^{1}(\Omega)\right)$ to the adjoint problem:

$$
\begin{aligned}
p_{t t} & =\Delta p+h p+\left(w_{\beta}-z\right) \chi_{Q^{\prime}}, \quad \text { in } Q \\
p & =0, \quad \text { on } \partial \Omega \times(0, T) \\
p & =p_{t}=0, \quad t=T, x \in \Omega
\end{aligned}
$$

where $\chi_{Q^{\prime}}$ is the characteristic function of the set $Q^{\prime}$. Furthermore, $h_{\beta}$ satisfies

$$
h_{\beta}=\max \left(-M, \min \left(-\frac{w p}{\beta}, M\right)\right) .
$$

Proof: Let $h_{\beta}+\varepsilon \ell \in U$ for $\varepsilon>0$ and $w^{\varepsilon}=w\left(h_{\beta}+\varepsilon \ell\right)$. Since the minimum of $J_{\beta}$ is achieved at $h_{\beta}$, we have

$$
\begin{aligned}
0 & \leq \lim _{\varepsilon \rightarrow 0^{+}} \frac{J_{\beta}\left(h_{\beta}+\varepsilon \ell\right)-J_{\beta}\left(h_{\beta}\right)}{\varepsilon} \\
& =\int_{Q^{\prime}} \Psi\left(w_{\beta}-z\right) d x d t+\beta \int_{Q} h_{\beta} \ell d x d t .
\end{aligned}
$$

Let $p$ be the weak solution of the adjoint problem (2.2). Using (2.1) and (2.2) in (2.4), we obtain

$$
0 \leq \int_{Q} \ell\left(p w_{\beta}+\beta h_{\beta}\right) d x d t
$$

which gives the desired characterization (2.3).

Thus, for a fixed $\beta$, the optimal control can be expressed from (2.3) in terms of the solution of the optimality system

$$
\begin{array}{rlrl}
w_{t t} & =\Delta w+\max \left(-M, \min \left(-\frac{w p}{\beta}, M\right)\right) w+f \text { in } Q \\
p_{t t}=\Delta p+\max \left(-M, \min \left(-\frac{w p}{\beta}, M\right)\right) p+(w-z) \chi_{Q^{\prime}} & \text { in } Q \\
w & =p=0 & \text { on } \partial \Omega \times(0, T) \\
w & =w_{0}, \quad w_{t}=w_{1} & t=0, x \in \Omega \\
p & =p_{t}=0 & t=T, x \in \Omega .
\end{array}
$$


Note that bounded solutions of the optimality system are unique if $T$ is sufficiently small. See [4], [6] for similar results. If one assumes $h$ is a function of $x$ only or $t$ only, better uniqueness results can be obtained [6].

\section{IDENTIFICATION PROBLEM}

We now use a sequence of optimal controls (as $\beta \rightarrow 0$ ) to identify $h$ from observations $z$. We do not assume that $z$ is in the range of the map

$$
\left.h \in U \rightarrow w(h)\right|_{Q^{\prime}}
$$

This situation could occur from inaccurate observations.

Theorem 3.1: There exist: (i) a sequence $\beta_{n} \rightarrow 0,($ ii $)$ corresponding optimal controls, $h_{\beta_{n}}$, for the functional $J_{\beta_{n}}(h),(i i i) h^{*} \in U$, and (iv) $w^{*}=w\left(h^{*}\right)$ (solution of (1.1)), such that

$$
\begin{array}{r}
h_{\beta_{n}} \rightarrow h^{*} \text { weakly in } L^{2}(Q), \\
w_{\beta_{n}}=w\left(h_{\beta_{n}}\right) \rightarrow w^{*} \text { weakly in } L^{2}\left(0, T ; H_{0}^{1}(\Omega)\right),
\end{array}
$$

and

$$
\int_{Q^{\prime}}\left(w^{*}-z\right)^{2} d x d t=\inf _{h \in U} \int_{Q^{\prime}}(w(h)-z)^{2} d x d t .
$$

Note: The limit $w^{*}$ can be interpreted as a (not necessarily unique) projection of $z$ onto the range of the map (3.1).

Proof: Note that the a priori estimates from Lemma 1.1 are independent of $\beta$. Thus we can find a sequence $\beta_{n} \rightarrow 0$ and $h^{*} \in U, w^{*} \in L^{2}\left(0, T ; H_{0}^{1}(\Omega)\right)$ such that

$$
J_{\beta_{n}}\left(h_{\beta_{n}}\right)=\inf _{h \in U} J_{\beta_{n}}(h)
$$

and

$$
\begin{array}{r}
h_{\beta_{n}} \rightarrow h^{*} \text { weakly in } L^{2}(Q), \\
w_{\beta_{n}} \rightarrow w^{*} \text { weakly in } L^{2}\left(0, T ; H_{0}^{1}(\Omega)\right), \\
w_{\beta_{n}} \rightarrow w^{*} \text { strongly in } L^{2}(Q), \\
\left(w_{\beta_{n}}\right)_{t t} \rightarrow\left(w^{*}\right)_{t t} \text { weakly in } L^{2}\left(0, T ; H^{-1}(\Omega)\right) .
\end{array}
$$

where $w^{*}=w\left(h^{*}\right)$, is the weak solution of (1.1). Now for any $\bar{h} \in U, \bar{w}=w(\bar{h})$, we have

$$
J_{\beta}\left(h_{\beta}\right) \leq J_{\beta}(\bar{h})
$$


Letting $\beta_{n} \rightarrow 0$ in (3.3) gives

$$
\int_{Q^{\prime}}\left(w^{*}-z\right)^{2} d x d t \leq \int_{Q^{\prime}}(\bar{w}-z)^{2} d x d t
$$

which yields the desired result (3.3).

\section{Acknowledgments}

S.L and V.P. acknowledge partial support from the US Department of Energy, Office of Basic Energy Sciences under contract No. ACO5-96OR22464 with Lockheed Martin Energy Research Corporation.

\section{References}

[1] Banks, H.T. and Kunisch, K., Estimation Techniques for Distributed Parameter Systems, Birkhäuser, Boston, 1989

[2] Borggaard, J. and Burns, J., A PDE Sensitivity Equation Method for Optimal Aerodynamic Design, ICASE Report 96-44, NASA Langley Research Center

[3] Evans, L.C., Partial Differential Equations, AMS, Providence, 1998

[4] Lenhart, S., Protopopescu, V., and Yong J., "Optimal Control of a Reflection Boundary Coefficient in an Acoustic Wave Equation", Applicable Analysis, 68(1998), 179-194

[5] Lenhart, S., Protopopescu, V., and Yong J., "Solving Inverse Problems of Identification Type by Optimal Control Methods", in Applied Nonlinear Dynamics and Stochastic Systems near the Millenium, J.B. Kadtke and A. Bulsara, eds., American Institute of Physics, 1998, 87-94

[6] Liang, M., "Bilinear Optimal Control of a Wave Equation", University of Tennessee PhD Dissertation, 1998, and Mathematical Models and Methods in Applied Sciences, 9(1), 1999.

[7] Simon, J., "Compact Sets in The Space $L^{p}(0, T ; B)$ " , Annali di Matematica Pura ed Applicata CXLVI (1987), 65-96 